\title{
Primeradicals in Ternary Semi Groups
}

\author{
Seetha Mani. P, Sarala.Y, Jaya Lalitha. G
}

\begin{abstract}
In this paper we study the properties of prime radical of an ideal in a ternarysemigroup. We characterize different classes of ternarysemigroups by their properties of their radicals and nilpotent. We introduced and charaterize the notions of radical ideal generated by $P$ in ternarysemigroups.
\end{abstract}

\section{INTRODUCTION}

The literature of ternary algebraic system was introduced by D.M.Lehmer in 1932. The notion of ternarysemigroups was known to $\mathrm{S}$. Banach.

He showed by an example that a ternarysemigroup does not necessarily reduce to an ordinary semigroup. Bindu [2] developed the properties of prime and maximal ideals in ternarysemigroups.

Some significant results are given in [3,4].

\section{MAIN RESULT}

Definition 2.1: Let $P$ be an ideal of a ternarysemigroup $T$. Then primeradical of $\mathrm{P}$, symbolized by $\beta(\mathrm{P})$ is defined as P\}.

$\beta(P)=\{\cap$ of all primeideals of $T$ each of which contains

Definition 2.2: Let $\mathrm{T}$ be a Ternarysemigroup and $\mathrm{Q}$ be an ideal of $T$. Then $Q$ is called nilpotent ideal if $Q^{2 n+1}=0$ for some $n \in Z$ and $n \geq 0$.

Theorem 2.3: For an ideal $\mathrm{P}$ of a ternary semigroup $\mathrm{T}$. Then

(a) $\mathrm{P} \subseteq \beta(\mathrm{P})$.

(b) If $\mathrm{A}$ is a primeideal of $\mathrm{T}$ then $\mathrm{P} \subseteq \mathrm{A}$ if and only if $\beta(\mathrm{P}) \subseteq \mathrm{A}$.

(c) $\beta(P) \subseteq \beta(Q)$ where $Q$ is an ideal in T satisfying the condition $\mathrm{P} \subseteq \mathrm{Q}$.

(d) $\beta(\mathrm{P})$ is a semi primeideal of $\mathrm{T}$.

(e) $\beta(\mathrm{P})=\beta\left(\mathrm{P}^{2 \mathrm{n}+1}\right) ; \mathrm{n} \in \mathrm{Z}$ and $\mathrm{n} \geq 0$.

(f) Every nilpotent element of $\mathrm{T}$ is contained in $\beta(\mathrm{P})$

(g) $\beta(\beta(\mathrm{P}))=\beta(\mathrm{P})$.

Proof: By the definition of primeradical we can easily prove (a), (b), (c).

(d) We know that $\beta(P)$ is an ideal of $T$.. Let $\mathrm{R}^{3} \subseteq \beta(\mathrm{P})$; where $\mathrm{R}$ is an ideal of $\mathrm{T}$.

Now $\beta(P)=\cap\left\{A_{i} / P \subseteq A_{i}, A_{i}\right.$ is a primeideal in $\left.T\right\}$. So $R^{3} \subseteq$ $\mathrm{A}_{\mathrm{i}}, \forall \mathrm{A}_{\mathrm{i}}$. Then $\mathrm{A}_{\mathrm{i}}$ is prime; $\mathrm{R} \subseteq \mathrm{A}_{\mathrm{i}}, \forall \mathrm{A}_{\mathrm{i}}$. Therefore $\mathrm{R} \subseteq \beta(\mathrm{P})$. Hence $\beta(P)$ is a semi primeideal of $T$.

(e) $\mathrm{P}$ is an ideal in $\mathrm{T}, \mathrm{P}^{2 \mathrm{n}+1} \subseteq \mathrm{P} ; \mathrm{n} \in \mathrm{Z}$ and $\mathrm{n} \geq 0$. Thus by (c), $\beta\left(\mathrm{P}^{2 \mathrm{n}+1}\right) \subseteq \beta(\mathrm{P})$.

Supoose $x \in \beta(P)$. Now $\beta(P)=\bigcap\left\{A_{i} / P \subseteq A_{i}, A_{i}\right.$ is prime ideal in $\mathrm{T}\}$.

Revised Manuscript Received on April 12, 2019.

Seetha Mani. P, Department of Mathematics, Koneru Lakshmaiah Education Foundation, Vaddeswaram, Guntur, A.P, India

Sarala.Y, Faculty of Mathematics, National Institute of Technology, Tadepalligudem, A.p, India

Jaya Lalitha G, Department of Mathematics, Koneru Lakshmaiah Education Foundation, Vaddeswaram, Guntur, A.P, India
Then $x \in A_{i} \forall A_{i}$. Suppose we assume that $x \notin \beta\left(P^{2 n+1}\right)$. Then $\exists$ a primeideal $\mathrm{B} \in \mathrm{T} \ni \mathrm{B} \supseteq \mathrm{P}^{2 \mathrm{n}+1}$ and $\mathrm{x} \notin \mathrm{B}$. Since $\mathrm{B}$ is prime, $\mathrm{P}^{2 \mathrm{n}+1} \subseteq \mathrm{B} \Rightarrow \mathrm{P} \subseteq \mathrm{B}$ whence $\mathrm{B}$ is some $\mathrm{A}_{\mathrm{i}}$, Which is a contradiction. Hence $x \in \beta\left(\mathrm{P}^{2 \mathrm{n}+1}\right)$. Hence $\beta(\mathrm{P})=\beta\left(\mathrm{P}^{2 \mathrm{n}+1}\right)$.

(f) Let $\mathrm{M}$ be the nilpotentideal in $\mathrm{T}$. Then $\mathrm{M}^{2 \mathrm{n}+1}=\{0\}$ for some $n \in Z$ and $n \geq 0$. Hence $\mathrm{M}^{2 \mathrm{n}+1} \subseteq \beta(\mathrm{P})$. Further $\mathrm{M}^{2 \mathrm{n}+1} \subseteq$ $\mathrm{A}_{\mathrm{i}} \forall \mathrm{A}_{\mathrm{i}} \supseteq \mathrm{P}$ and $\mathrm{A}_{\mathrm{i}}$ is a primeideal. Then $\mathrm{M} \subseteq \mathrm{A}_{\mathrm{i}} \forall \mathrm{A}_{\mathrm{i}}$. Therefore $\mathrm{M} \subseteq \beta(\mathrm{P})$.

(g) By (a); $\mathrm{P} \subseteq \beta(\mathrm{P})$. By (c) $\beta(\mathrm{P}) \subseteq \beta(\beta(\mathrm{P}))$. Let $\mathrm{x} \in$ $\beta(\beta(\mathrm{P}))$ and $\left\{\mathrm{A}_{\mathrm{i}}\right\}_{\mathrm{i} \in \mathrm{I}}$ be the group of primeideals in $\mathrm{T} \ni \mathrm{P} \subseteq \mathrm{A}_{\mathrm{i}}$ $\forall \mathrm{i} \in \mathrm{I}$. By the definition $\beta(\mathrm{P}) \subseteq \mathrm{A}_{\mathrm{i}} \forall \mathrm{i} \in \mathrm{I}$. Whence $\beta(\beta(\mathrm{P})) \subseteq$ $A_{i}$. Thus $x \in A_{i} \forall i \in I$. Whence $x \in \beta(P)$. Hence $\beta(\beta(P))=$ $\beta(\mathrm{P})$.

Theorem 2.4: [4] Let $\mathrm{P}$ be an ideal in a ternarysemigroup $\mathrm{T}$. Then $\beta(\mathrm{P})=\{\mathrm{t} \in \mathrm{T} /$ every $\mathrm{m}$-system in $\mathrm{T}$ which contains $\mathrm{t}$, has a nonempty intersection with $\mathrm{P}\}$.

Proposition 2.5: Let $\mathrm{P}$ be an ideal in a ternarysemigroup T. If $x \in \beta(P)$ then $\exists$ an integer $n \geq 0 \exists x^{2 n+1} \in P$.

Proof: Let $x \in \beta(P)$. Then by theorem 2.4 , every $m$ - system in $T$ containing $x$ has a nonempty intersection with $P$. Consider $M=\left\{x^{2 n+1} / n \in Z\right.$ and $\left.n \geq 0\right\}$. Then $M$ is an $m$ system containing $\mathrm{x}$. Therefore $\mathrm{M} \cap \mathrm{P} \neq \emptyset$. Then $\exists$ an integer $\mathrm{n} \geq 0 \ni \mathrm{x}^{2 \mathrm{n}+1} \in \mathrm{P}$.

Proposition 2.6: Suppose $\mathrm{T}$ is a commutative ternarysemigroup and $\mathrm{M}$ is an $\mathrm{m}$-system in $\mathrm{T}$ which contains $\mathrm{x}$. Then $\exists$ an integer $\mathrm{n} \geq 0 \exists \mathrm{x}^{2 \mathrm{n}+1} \mathrm{ab} \in \mathrm{M}$ where $\mathrm{a}, \mathrm{b} \in \mathrm{T}$.

Proof: We recall the definition of commutativity and m-system of $T$. Since $x \in M, \exists a_{1}, a_{2}, a_{3}, a_{4}$ in $T \exists x_{1} x_{2} x \in M$ or $\mathrm{xa}_{1} \mathrm{a}_{2} \mathrm{xa}_{3} \mathrm{a}_{4} \mathrm{x} \in \mathrm{M}$ or

$x a_{1} a_{2} x a_{3} x a_{4} \in M$ or $a_{1} x a_{2} x a_{3} a_{4} x \in M$. This will imply that $x\left(a_{1} x a_{2}\right) x \in M$ or $T$ being commutative, $x^{3} a_{1} a_{2} \in M$ or $x^{3} a_{1} a_{2} a_{3} a_{4} \in M$.

Let $\mathrm{x}^{3} \mathrm{a}_{1} \mathrm{a}_{2} \in \mathrm{M}$. Then $\exists \mathrm{a}_{5}, \mathrm{a}_{6}, \mathrm{a}_{7}, \mathrm{a}_{8} \in \mathrm{T} \ni \mathrm{x}^{5} \mathrm{a}_{1} \mathrm{a}_{2} \mathrm{a}_{5} \mathrm{a}_{6} \in \mathrm{M}$ or $x^{5} a_{1} a_{2} a_{5} a_{6} a_{7} a_{8} \in M$.

Let $x^{3} a_{1} a_{2} a_{3} a_{4} \in M$. Then $\exists b_{1}, b_{2}, b_{3}, b_{4} \in T \exists x^{5} a_{1} a_{2} a_{3} a_{4} b_{1} b_{2}$ $\in M$ or $x^{5} a_{1} a_{2} a_{3} a_{4} b_{1} b_{2} b_{3} b_{4} \in M$. Proceeding in this process we get for every integer $n \geq 0, x^{2 n+1} a b \in M$ for some $a, b \in T$.

Proposition 2.7: Suppose $\mathrm{P}$ be an ideal in a commutative ternarysemigroup $\mathrm{T} \ni \mathrm{x}^{2 \mathrm{n}+1} \in \mathrm{P}$, where $\mathrm{x} \in \mathrm{T}, \mathrm{n} \in \mathrm{Z}$ and $\mathrm{n} \geq 0$. Then $x \in \beta(P)$.

Proof: Let $M$ be any m-system in $T$ and $x \in T$. Then by above Proposition 2.6, $\mathrm{x}^{2 \mathrm{n}+1} \mathrm{ab} \in \mathrm{M}$, for some $\mathrm{a}, \mathrm{b} \in \mathrm{T}$. As $\mathrm{P}$ is an ideal and $x^{2 n+1} \in P, x^{2 n+1} a b \in P$. So $M \cap P \neq \emptyset$. Therefore by Theorem 2.4, $x \in \beta(P)$.

By above Propositions 2.5 and 2.7 we prove the following theorem.

Theorem 2.8: Let $\mathrm{T}$ be a commutative ternarysemigroup and $\mathrm{P}$ be an ideal of $\mathrm{T}$. Then $\beta(\mathrm{P})=\left\{\mathrm{x} \in \mathrm{T} / \mathrm{x}^{2 \mathrm{n}+1} \in \mathrm{P}\right.$ for some $\mathrm{n} \in \mathrm{Z}^{+}$and $\left.\mathrm{n} \geq 0\right\}$ 
Definition 2.9: An ideal $P$ in a ternarysemigroup $T$ is called a prime radicalideal if $\beta(P)=P$.

Prime radicalideal simply called as a radicalideal.

Proposition 2.10: The consecutive conditions in an ideal $\mathrm{P}$ of a ternarysemigroup $\mathrm{T}$ are identical:

(a) $\beta(P)=P$

(b) $x^{2 n+1} \in P \Rightarrow x \in P$, where $n \in Z$ and $n \geq 0$.

Proof: $(\mathrm{a}) \Rightarrow$ (b). Let $\mathrm{x}^{2 \mathrm{n}+1} \in \mathrm{P}$ then by above Proposition 2.7, $x \in \beta(P)=P \Rightarrow x \in P$.

(b) $\Rightarrow$ (a). We have $P \subseteq \beta(P)$. Let $t \in \beta(P)$. Then by above Proposition $2.5 \exists$ an integer $n \geq 0 \ni t^{2 n+1} \in P$. Hence by (b)

$t \in \mathrm{P}$. Hence $\beta(\mathrm{P}) \subseteq \mathrm{P}$. Therefore $\beta(\mathrm{P})=\mathrm{P}$.

Theorem 2.11: In a ternarysemigroup intersection of any

set of radicalideals is a radicalideal.

Proof: Let $\mathrm{T}$ be a ternarysemigroup and $\left\{\mathrm{S}_{\mathrm{i}} / \mathrm{i} \in \Lambda\right.$ be any set of radicalideals in $\mathrm{T}$. Then by above definition $2.9, \beta\left(\mathrm{S}_{\mathrm{i}}\right)=$ $\mathrm{S}_{\mathrm{i}}$. Now $\cap \mathrm{S}_{\mathrm{i}} \subseteq \mathrm{S}_{\mathrm{i}} \forall \mathrm{i} \in \Lambda$. So by above proposition 2.3 (c), $\beta\left(\bigcap_{i \in \Lambda}^{S_{i}}\right) \subseteq$ Si $\forall \mathrm{i} \in \Lambda$. Therefore $\beta\left(\bigcap_{i \in \Lambda} S_{i}\right) \subseteq \bigcap_{i \in \Lambda} S_{i} \forall \mathrm{i} \in \mathrm{A}$. Again $\bigcap_{i \in \Lambda} S_{i} \subseteq \beta\left(\bigcap_{i \in \Lambda} S_{i}\right.$ ) (by proposition 2.3(a)). Therefore $\beta($ $\left.\bigcap_{i \in \Lambda} S_{i}\right)=\bigcap_{i \in \Lambda} S_{i} \forall \mathrm{i} \leq \mathrm{i} \in \Lambda$. Hence $\bigcap_{i \in \Lambda} S_{i}$ is a radicalideal

Definition 2.12: Let $T$ is a ternarysemigroup with a subsemigroup $\mathrm{P}$ and for an ideal $\mathrm{I}, \mathrm{A}=\mathrm{I} \cap \mathrm{P}$ is an ideal. If there is some other ideal $\mathrm{J} \in \mathrm{T} \ni \mathrm{I} \subseteq \mathrm{J}$ and $\mathrm{A}=\mathrm{J} \cap \mathrm{P}$ then we say that $\mathrm{I}$ can be extended to an ideal in $\mathrm{T}$ which will also contracts to A.

Theorem 2.13: Suppose $\mathrm{P}$ be an $m$-system and $\mathrm{N}$ be an ideal of a ternarysemigroup $\mathrm{T} \ni \mathrm{N} \cap \mathrm{P}=\emptyset$. Then $\exists$ a maximalideal $\mathrm{M}$ of $\mathrm{T}$ contained $\mathrm{N} \ni \mathrm{M} \cap \mathrm{P}=\emptyset$. Further, $\mathrm{M}$ is a primeideal of $\mathrm{T}$.

Theorem 2.14: Let $\mathrm{T}$ be a commutative ternarysemigroup and $\mathrm{P}$ be a ternary subsemigroup of $\mathrm{T}$. Let $\mathrm{I}$ be a radicalideal of $\mathrm{T} \ni \mathrm{xyz} \in \mathrm{I}, \mathrm{x} \in \mathrm{P} ; \mathrm{y}, \mathrm{z} \in \mathrm{T}$ implies that either $\mathrm{x} \in \mathrm{I}$ or $\mathrm{y} \in$ I or $\mathrm{z} \in \mathrm{I}$. Then $\mathrm{A}=\mathrm{I} \cap \mathrm{P}$ is a primeideal in P. Moreover I can be declared as an intersection of primeideals each of which contradicts to A.

Proof \& Results: Suppose $x, y, z \in P \ni x y z \in A$. Then xyz $\in \mathrm{I}$. Therefore by our assumption either $\mathrm{x} \in \mathrm{I}$ or $\mathrm{y} \in \mathrm{I}$ or $\mathrm{z} \in \mathrm{I}$. Thus either $\mathrm{x} \in \mathrm{A}$ or $\mathrm{y} \in \mathrm{A}$ or $\mathrm{z} \in \mathrm{A}$. So $\mathrm{A}$ becomes a primeideal. Put $X=\{\mathrm{J}: \mathrm{J}$ is a primeideal of T with $\mathrm{J} \supseteq \mathrm{I}$ and $\mathrm{J}$ $\cap \mathrm{P}=\mathrm{A}\}$. Then $\mathrm{I} \subseteq \mathrm{X}$. Now we prove $\mathrm{X} \subseteq \mathrm{I}$. Let $\mathrm{x} \notin \mathrm{I}$. Then the m-system $m=\{y\} \cup\left\{d y^{2 n} / d \in P\right.$ but $d \notin A$ and $\left.n \in Z^{+}\right\}$ and $\mathrm{m} \cap \mathrm{I}=\varnothing$. Then by proposition $2.13 \exists$ a maximalideal $\mathrm{B} \supseteq$ I with $B \cap M=\varphi$ which is again prime.

Then $\mathrm{A} \subseteq \mathrm{B} \cap \mathrm{P}$. Again for $\mathrm{b} \in \mathrm{B} \cap \mathrm{P}$, by $^{2} \in \mathrm{B}$; $\mathrm{B}$ is an ideal of T. It follows that $b y^{2} \notin \mathrm{M}$. This is together with the definition of $\mathrm{M}$ and $\mathrm{b} \in \mathrm{P}$ implies that $\mathrm{b} \in \mathrm{A}$. Therefore $\mathrm{B} \cap \mathrm{P}$ $\subseteq \mathrm{A}$. Hence $\mathrm{A}=\mathrm{B} \cap \mathrm{P}$. Again $\mathrm{y} \notin \mathrm{B}$ as $\mathrm{y} \in \mathrm{M}$ and $\mathrm{M} \cap \mathrm{B}=$ $\varphi$. Therefore $\mathrm{y} \notin \mathrm{X}$ and $\mathrm{X} \subseteq \mathrm{I}$. Hence $\mathrm{I}=\mathrm{X}$.

Definition 2.15: Let $T$ be a ternarysemigroup and $\mathrm{P} \subseteq \mathrm{T}$ for some $\mathrm{P}$. Let $\{\mathrm{P}\}$ be the radicalideal generated by $\mathrm{P}$ and is defined as the intersection of all radicalideals of $\mathrm{T}$ such that each ideal contains P. Clearly $\{P\}$ is the smallest radicalideal that contains $\mathrm{P}$. We denote $\{\mathrm{P}, \mathrm{x}\}$ as $\{\mathrm{P} \cup\{\mathrm{x}\}\}$.

Theorem 2.16: In a commutative ternarysemigroup $T$ satisfying ascending chain condition on radicalideal can be expressed as the finite intersection of primeideals.

Proof: Let $\mathrm{T}$ be a commutative ternarysemigroup satisfying ascending chain condition on radicalideals. Put $\mathrm{R}$ is the set of all radicalideals which cannot be expressed as the finite intersection primeideals and $\mathrm{R} \neq \varnothing$. $\mathrm{T}$ satisfies ascending chain condition on radicalideals, $\mathrm{R}$ has a maximal element say I. Since I $\in$ R and it cannot be expressed as the finite intersection of primeideals, i.e I is not prime.

Therefore $\exists \mathrm{x}, \mathrm{y}, \mathrm{z} \in \mathrm{S} \ni \mathrm{xyz} \in \mathrm{I}$ but $\mathrm{x} \notin \mathrm{I}, \mathrm{y} \notin \mathrm{I}, \mathrm{z} \notin \mathrm{I}$.

Then each of the radicalideals $\{\mathrm{I}, \mathrm{x}\},\{\mathrm{I}, \mathrm{y}\},\{\mathrm{I}, \mathrm{z}\} \supseteq \mathrm{I}$. Therefore each of them can be expressed as the finite intersection of primeideals in $\mathrm{T}$.

Now $\{\mathrm{I}, \mathrm{x}\},\{\mathrm{I}, \mathrm{y}\},\{\mathrm{I}, \mathrm{z}\} \subseteq \mathrm{I},\{\mathrm{xyz}\} \subseteq \quad \mathrm{I}$. For any $\mathrm{b}$ $\in\{I, x\} \cap\{I, y\} \cap\{I, z\}, b^{3} \in I \Rightarrow b \in I$ as $I$ is a radicalideal. So $\{\mathrm{I}, \mathrm{x}\} \cap\{\mathrm{I}, \mathrm{y}\} ;\{\mathrm{I}, \mathrm{z}\} \subseteq$ I. Clearly $\mathrm{I} \subseteq\{\mathrm{I}, \mathrm{x}\} \cap\{\mathrm{I}, \mathrm{y}\} \cap\{\mathrm{I}, \mathrm{z}\}$. Therefore $\mathrm{I}=\{\mathrm{I}, \mathrm{x}\} \cap\{\mathrm{I}, \mathrm{y}\} \cap\{\mathrm{I}, \mathrm{z}\}$. Hence I can be expressed as the finite intersection of primeideals, which is a contradiction. Therefore $\mathrm{R}=\emptyset$. Hence the proof.

\section{REFERENCES}

1. Anjaneyulu.A, "Semigroups in which prime ideals are maxima"l, Semigroup Forum, 22 (1981), 151-158.

2. Bindu.P, Sarala.Y, "Prime and maximal ideals in ternary semigroup", Indian Journal of Science and Technology, 8, No-14,(2015), 1-4.

3. Jaya lalitha, G. Sarala, Y., "On ternary semigroups which are unions of a finite number of principal ideals", Global journal of Pure and Applied Mathematics, Vol. 11, No.2, (2015), 1079-1086.

4. Seethamani.P, Sarala.Y, "Strongly prime radicals of a ternary semigroups", IJPAM, 116, No-1, (2017), 169-175. 